\title{
Die Naturheilkunde in Forschung und Lehre: Neue Perspektiven?
}

Nach einem längeren Anlauf wurde vom Bundesratsplenum am 26. April 2002 mit grosser Mehrheit die Reform (9.Novelle) der Approbationsordnung für Ärzte verabschiedet. Das Medizinstudium kann damit zum Wintersemester 2003/2004 nach der neuen Ausbildungsordnung begonnen werden. Die neue Approbationsordnung soll eine der umfangreichsten Reformen des Medizinstudiums seit 1970 bewirken, Hauptziel ist die Realisierung eines stärkeren Praxisbezuges im Studium sowie die deutlichere interdisziplinäre Gestaltung des vermittelten Behandlungsprozesses auf der Grundlage einer ganzheitlichen Sicht der Patientensituation. In diesem $\mathrm{Zu}$ sammenhang sollen Prävention, Gesundheitsförderung und Allgemeinmedizin besser in den Unterricht einbezogen werden.

Für den Bereich der Naturheilkunde wesentlich erscheint die Empfehlung zur Modifikation des §27. Der Ausschuss empfiehlt Naturheilverfahren, Physikalische Medizin und Rehabilitation stärker in der Ausbildung mit zu berücksichtigen.

«Begründung: unabhängig von der fachlichen Bewertung werden Naturheilverfahren von den Patienten in hohem Masse nachgefragt. Daher ist es erforderlich, dass der Arzt - unabhängig von seiner späteren fachlichen Ausrichtung - Grundkenntnisse in Naturheilverfahren besitzt und diese Verfahren beurteilen kann. Grundkenntnisse in den Naturheilverfahren sollen daher zu den Zulassungsvoraussetzungen zum zweiten Abschnitt der ärztlichen Prüfung gehören.»

Insgesamt soll aber auch die Qualität der Lehre in Deutschland verbessert werden. Der Gesundheitsausschuss im Bundesrat geht hierbei davon aus, dass der Lehre an deutschen medizinischen Fakultäten insgesamt zu wenig Beachtung geschenkt wird. In Zukunft sollen nun Lehrveranstaltungen regelmässig auf ihren Erfolg hin evaluiert und die Ergebnisse öffentlich gemacht werden.

Erfreulicherweise ist damit die universitäre Ausbildung im Bereich Naturheilverfahren und der Leistungsnachweis gesetzlich fest verankert.
Die Empfehlungen des Bundesausschusses greifen letztlich eine seit langem in Deutschland erkennbare Entwicklung auf. So wurde bei der Vorstellung der demoskopischen Studie «Naturheilmittel 2002» erneut aufgezeigt, dass ein steigender Bevölkerungsanteil im Krankheitsfalle auch auf natürliche Heilmittel vertraut. Bei der Vorstellung der Studie am 16.04.2002 forderte die Direktorin der Allensbach Institute, Frau Prof. Noelle Naumann, die Einrichtung von insgesamt 10 Lehrstühlen im Bereich Naturheilkunde, um die zukünftigen Ärzte angemessen informieren zu können. Dies ist wohl zum aktuellen Zeitpunkt schon allein aufgrund mangelnder personeller Ressourcen nicht adäquat realisierbar. Eine beeindruckend rascher Entwicklungsprozess konnte in diesem $\mathrm{Zu}$ sammenhang in den vergangenen Jahren in den USA beobachtet werden. Zeitnah mit der Postulierung eines zukünftig mehr patientengesteuerten medizinischen Versorgungssystems durch die Harvard-Ökonomin Regina Herzlinger ( «Consumer-driven health care») wurden die Erhebungen von David Eisenberg zur Verbreitung naturheilkundlicher und komplementärer Verfahren in der amerikanischen Bevölkerung im New England Journal of Medicine und im JAMA publiziert. Und während noch 1993 im New England Journal of Medicine «CAM» (Complementary and Alternative Medicine) als «Methods not taught at medical schools» definiert wurde, war bereits 1999 in 75\% aller amerikanischen medizinischen Fakultäten CAM in der medizinischen Ausbildung zumindest partiell implementiert. Es bleibt abzuwarten, ob die Patientennachfrage in Deutschland eine ähnliche universitäre Dynamik bewirken kann.

Auch unter den deutschen Medizinstudenten findet sich inzwischen ein wachsendes Interesse an wissenschaftlich fundierten Informationen über Naturheilkunde und Ihre Verfahren. Einen vielversprechenden Eindruck von dieser Entwicklung geben die ersten Erfahrungen der naturheilkundlichen Vorlesung an der Universität Essen. 
Die Abteilung für Innere Medizin V, Naturheilkunde und Integrative Medizin an den Kliniken Essen-Mitte führt seit $2 \mathrm{Se}-$ mestern eine evaluierte Vorlesung mit Blockpraktikum im Bereich Naturheilkunde durch. Diese freiwillige und zertifizierte Veranstaltung findet turnusmässig an Samstagen statt. Trotz der starken zeitlichen Belastung durch Pflichtpraktika zeichnete sich hier ein wachsendes Interesse an den Veranstaltungen ab. Nach initial 30 festen Teilnehmern während aller Veranstaltungen im letzten Semester nahmen jetzt 50-60 Studenten an den mehrstündigen Veranstaltungen teil. In der ausführlichen begleitenden Evaluation wurden die Themenbereiche und der Informationsgehalt durchweg als gut bis sehr gut beurteilt. Aus der Evaluation geht aber auch hervor, dass für die teilnehmenden Studenten primär die evidenzbasierten und wissenschaftlich fundierten Naturheilverfahren von Interesse sind. Spekulative oder esoterische Aspekte und Verfahren, auch wenn sie sehr bekannt sind, werden hingegen als weniger interessant bewertet. Insbesondere die klassischen Verfahren Ernährung, Ordnungstherapie und Hydrotherapie, aber auch die Akupunktur erfahren eine grosse Akzeptanz. Interessanterweise sind auch die präventiven Aspekte für die Studenten bedeutsam. Beispielsweise sind $70 \%$ der befragten Studenten an der Vermittlung einer strukturierten Raucherentwöhnungspraxis interessiert. Für alle Themenbereiche aber gilt: Die kombinierte Vermittlung von Praxis, direkter Anleitung und klinisch wissenschaftlicher Evidenz gilt als wesentlich.
Die Realisierung einer qualitativ hochwertigen Vorlesung mit ausreichend praktischer Wissensvermittlung ist aber auch mit personellen Aufwand verbunden. So wurden im Rahmen dieser Vorlesung praktische Demonstrationen und «Bed-side-teaching» mit Bindegewebsdiagnostik, Blutegeltherapie und Akupunktur durchgeführt, Workshops boten Einführungen in Entspannungsverfahren, Hydrotherapie, Massagetechniken, Phytotherapie sowie in mediterraner Vollwerternährung mittels Lehrküche an.

Neben der insgesamt sehr positiven Resonanz auf die Vorlesung zeigten sich erfreulicherweise auch mehr als $50 \%$ der befragten teilnehmenden Studenten stark an einer naturheilkundlich ausgerichteten Doktorarbeit interessiert.

Somit unterstützt die Approbationsnovelle eine erfreuliche Entwicklung an den medizinischen Fakultäten. Vermutlich ergänzen sich der Wunsch der Studenten nach einer praxisorientierten patientennahen Ausbildung und die personotrope patientenorientierte Herangehensweise der Naturheilkunde auf sinnvolle Weise. Unabdingbar ist es aber auch, dass sich die Naturheilkunde im Bereich der universitären Lehre hohe Qualitätsstandards setzt und die Lehrinhalte weiter standardisiert werden. Eine derartige Implementierung der Naturheilverfahren in die Lehre wird hoffentlich nicht ohne positiven Einfluss auf die Forschung und auch deren Unterstützung bleiben.

G. Dobos, A. Michalsen, Essen 